\title{
COVERAGE OF IMPLEMENTATIONS BY SIMULATING SPECIFICATIONS
}

\author{
Hana Chockler and Orna Kupferman \\ School of Engineering and Computer Science \\ Hebrew University \\ Jerusalem 91904, Israel \\ \{hanac,orna\}@cs.huji.ac.il
}

\begin{abstract}
In formal verification, we verify that an implementation is correct with respect to a specification. When verification succeeds and the implementation is proven to be correct, there is still a question of how complete the specification is, and whether it really covers all the behaviors of the implementation. In this paper we study coverage for simulation-based formal verification, where both the implementation and the specification are modelled by labeled state-transition graphs, and an implementation $\mathcal{I}$ satisfies a specification $\mathcal{S}$ if $\mathcal{S}$ simulates $\mathcal{I}$. Our measure of coverage is based on small modifications we apply to $\mathcal{I}$. A part of $\mathcal{I}$ is covered by $\mathcal{S}$ if the mutant implementation in which this part is modified is no longer simulated by $\mathcal{S}$. Thus, "mutation coverage" tells us which parts of the implementation were actually essential for the success of the verification. We describe two algorithms for finding the parts of the implementation that are covered by $\mathcal{S}$. The first algorithm improves a naive algorithm that checks the mutant implementations one by one by exploiting the significant overlaps among the mutant implementations. The second algorithm is symbolic, and it improves a naive symbolic algorithm by reducing the number of variables in the OBDDs involved. In addition, we compare our coverage measure with other approaches for measuring coverage.
\end{abstract}

Keywords: Model Checking, Simulation, Coverage.

\section{Introduction}

In formal verification, we verify the correctness of a finite-state implementation with respect to a desired behavior by checking whether a labeled state-transition graph that models the implementation satisfies a specification of this behavior, expressed in terms of a temporal logic formula or a more abstract labeled state-transition graph. Beyond being fully-automatic, an additional attraction of formal verification tools is their ability to accompany a negative answer to the correctness query by a counterexample to the satisfaction of the specification in the implementation (for a survey, see Clarke et al., 1999). On the other hand, when the answer to the correctness query is positive, most 
verification tools terminate with no further information to the user. Since a positive answer means that the implementation is correct with respect to the specification, this seems like a reasonable policy. In the last few years, however, there has been growing awareness of the importance of suspecting the implementation of containing an error also in the case verification succeeds. The main justification of such suspects are possible errors in the modeling of the implementation or of the behavior, and possible incompleteness in the specification.

There are various ways to look for possible errors in the modeling of the implementation or the behavior. One direction is to detect vacuous satisfaction of the specification [Beer et al., 1997; Kupferman and Vardi, 1999], where cases like antecedent failure [ Beaty and Bryant, 1994] make parts of the specification irrelevant to its satisfaction. For example, the specification $\varphi=A G$ (req $\rightarrow A F$ grant $)$ is vacuously satisfied in an implementation in which req is always false. A similar direction is to check the validity of the specification. Clearly, a specification that is valid or is vacuously satisfied suggests some problem. It is less clear how to check completeness of the specification. Indeed, specifications are written manually, and their completeness depends entirely on the competence of the person who writes them. The motivation for such a check is clear: an erroneous behavior of the implementation can escape the verification efforts if this behavior is not captured by the specification. In fact, it is likely that a behavior not captured by the specification also escapes the attention of the designer, who is often the one to provide the specification.

In simulation-based verification techniques, coverage metrics are used in order to reveal states that were not visited during the testing procedure (i.e., not "covered" by this procedure); see [Ho and Horowitz, 1996; Bergmann and Horowitz, 1999; Fallah et al., 1999] and others. These metrics are a useful way of measuring progress of the verification process. However, the same intuition cannot be applied to formal verification, as the process of formal verification may visit all states regardless their essence to the success of the verification process. We can say that in testing, a state is "uncovered" if it is not essential to the success of the testing procedure. A similar idea can be applied to formal verification, where a state is defined as "uncovered" if its labeling is not essential to the success of the verification process. This approach was first suggested by Hoskote et al., 1999. Low coverage can point to several problems. One possibility is that the specification is not complete enough to fully describe all the possible behaviors of the implementation. Then, the output of a coverage check is helpful in completing the specification. Another possibility is that the implementation contains redundancies. Then, the output of the coverage check is helpful in simplifying the implementation.

There are two different approaches to coverage in model checking, where the specification is given as a temporal logic formula. One approach, introduced in Katz et al., 1999, states that a well-covered implementation should closely resemble the reduced tableau of its specification. Thus the coverage criteria of Katz et al. are based on the analysis of the differences between the implementation and the tableau of its specification. In the full version we discuss this approach in more detail. Another approach, introduced in Hoskote et al., 1999, is to check the influence of small changes in the implementation on the satisfaction of the specification. This approach is inspired 
by the definition of mutation coverage in simulation-based verification [Dill, 1998]. For a given implementation, we can consider a set of mutants, each representing one small change in the original implementation. The specification covers a mutation in an implementation if it is not satisfied in the corresponding mutant. Formally, for an implementation $\mathcal{I}$, modeled as a labeled state-transition graph, a state $w$ in $\mathcal{I}$, and an observable signal $q$, the mutant implementation $\tilde{\mathcal{I}}_{w, q}$ is obtained from $\mathcal{I}$ by flipping the value of $q$ in $w$ (the signal $q$ corresponds to a Boolean variable that is true in $w$ if $w$ is labeled with $q$ and is false otherwise; when we say that we flip the value of $q$, we mean that we switch the value of this variable). For a specification $\varphi$ that is satisfied in $\mathcal{I}$ and an observable signal $q$, a state $w$ of $\mathcal{I}$ is $q$-covered by $\varphi$ if $\tilde{\mathcal{I}}_{w, q}$ does not satisfy $\varphi$. Indeed, this indicates that the value of $q$ in $w$ is crucial for the satisfaction of $\varphi$ in $\mathcal{I}$. It is easy to see that for each observable signal, the set of $q$-covered states can be computed by a naive algorithm that performs model checking of $\varphi$ in $\tilde{\mathcal{I}}_{w, q}$ for each state $w$ of $I$. The naive algorithm, however, is very expensive, and is useless for practical applications.

The approach of Hoskote et al., 1999 is followed by Chockler et al., 2001b, where two alternatives to the naive algorithm are presented for specifications in the branching time temporal logic CTL. The first algorithm is symbolic and computes the set of pairs $\left\langle w, w^{\prime}\right\rangle$ such that flipping the value of $q$ in $w^{\prime}$ falsifies $\varphi$ in $w$. The second algorithm improves the naive algorithm by exploiting overlaps in the many mutant implementations that we need to check. The "mutant approach" is also taken in Chockler et al., 2001a, which studies coverage by specifications that are given as formulas in the linear temporal logic LTL or by automata on infinite words. Chockler et al. suggest alternative definitions of coverage, which suit better the linear case, and presents two algorithms for LTL and automata-based specifications. Both algorithms can be relatively easily implemented on top of existing model-checking tools.

In this paper we study coverage in design and verification methods in which the specification is given as a labeled state-transition graph. Consider an implementation and a specification. Both describe possible behaviors of the system, but the specification is more abstract than the implementation [Abadi and Lamport, 1991]. This approach, of representing both specifications and implementation as labeled state-transition graphs, suggests a top-down method for design development, called hierarchical refinement [ Lam and Shankar, 1984; Kurshan, 1994]: starting with a highly abstract specification, we construct a sequence of behavior descriptions, each of which refers to its predecessor as a specification, and is thus less abstract than the predecessor. At each stage the current implementation is verified to satisfy its specification. Verifying that an intermediate implementation satisfies its specification leads to detection of errors in the design as soon as they are introduced. Likewise, measuring coverage of an intermediate implementation with respect to its specification would lead to early detection of low coverage.

There are several ways of defining what it means for an implementation $\mathcal{I}$ to satisfy a specification $\mathcal{S}$. The two main ones are trace-based and tree-based. The former requires each computation of $\mathcal{I}$ to correlate with some computation of $\mathcal{S}$, and the latter requires each computation tree embodied in $\mathcal{I}$ to correlate with some computation tree embodied in $\mathcal{S}$. The simplest definition of such correlation is equivalence with respect 
to the variables joint to $\mathcal{I}$ and $\mathcal{S}$, as the implementation is typically defined over a wider set of variables, reflecting the fact that it is more concrete than the specification. With this interpretation, trace-based verification corresponds to trace containment [ Kurshan, 1994], and tree-based verification corresponds to simulation [Milner, 1971].

Simulation has several theoretically and practically appealing properties. First, since the definition of simulation is local, checking whether $\mathcal{S}$ simulates $\mathcal{I}$ can be done efficiently [Milner, 1980; Henzinger et al., 1995] and a witnessing relation for simulation can be computed symbolically [McMillan, 1993; Henzinger et al., 1995]. Second, simulation implies trace containment, whose checking for nondeterministic specifications is PSPACE-complete [Meyer and Stockmeyer, 1972]. The computational advantage is so compelling as to make simulation useful also to researchers that favor the linear approach to specification: in automatic verification, simulation is widely used as a sufficient condition for trace containment [Cleaveland et al., 1993]; in manual verification, trace containment is most naturally proved by exhibiting local witnesses such as simulation relations or refinement mappings (a restricted form of simulation relations) [Lamport, 1983; Lynch and Tuttle, 1987; Lynch, 1996].

We apply mutation-based coverage to simulation and suggest efficient algorithms to measure coverage in simulation. As in Hoskote et al., 1999, for an implementation $\mathcal{I}$, a state $w$ in $\mathcal{I}$, and an observable signal $q$, we say that $w$ is $q$-covered by a specification $\mathcal{S}$ if $\tilde{\mathcal{I}}_{w, q}$ is not simulated by $\mathcal{S}$. Intuitively, $w$ is $q$-covered by $\mathcal{S}$ if flipping the value of $q$ in $w$ creates a behavior that is not permitted by $\mathcal{S}$. As in the context of model checking, the naive algorithm computes coverage by executing a simulation computation algorithm $|W|$ times, once for each mutant implementation. We suggest two algorithms that improve the naive algorithm. Our algorithms are built on top of algorithms that compute the simulation relation. The first algorithm is built on top of the enumerative simulation algorithm of Henzinger et al., 1995. The time complexity of the algorithm of Henzinger et al. is $O\left(m^{\prime} n+m n^{\prime}\right)$, where $m, n$, and $m^{\prime}, n^{\prime}$ are the sizes of transition relations and state spaces of the implementation and specification. To the best of our knowledge, this is the best time complexity known for the problem ${ }^{1}$. Our algorithm exploits similarities between the mutant implementations, and has an average running time of $O\left(\left(m^{\prime} n+m n^{\prime}\right) \log n\right)$, while in the worst case its complexity does not exceed the complexity of the naive algorithm, which is $O\left(\left(m^{\prime} n+m n^{\prime}\right) n\right)$. The second algorithm is symbolic, and it computes, given an implementation $\mathcal{I}$ with state space $W$ and a specification $\mathcal{S}$ with state space $W^{\prime}$, the following ternary relation.

$$
\mathcal{C}=\left\{\left\langle w, v, w^{\prime}\right\rangle: w, v \in W, w^{\prime} \in W^{\prime}, \text { and } w^{\prime} \text { simulates } w \text { in } \tilde{\mathcal{I}}_{v, q}\right\} .
$$

Thus, a triplet $\left\langle w, v, w^{\prime}\right\rangle$ is in $\mathcal{C}$ iff $w^{\prime}$ simulates $w$ in the mutant implementation $\tilde{\mathcal{I}}_{v, q}$, obtained from $\mathcal{I}$ by flipping the value of $q$ in $v$. In particular, a state $v$ is $q$-covered iff there exists an initial state $w_{0}$ of $\mathcal{I}$ such that for all initial states $w_{0}^{\prime}$ of $\mathcal{S}$ we have $\left\langle w_{0}, v, w_{0}^{\prime}\right\rangle$ not in $\mathcal{C}$, in which case $\mathcal{S}$ does not simulate $\tilde{\mathcal{I}}_{v, q}$. A naive implementation of Milner's fixed-point expression for simulation requires $2\left(n+n^{\prime}\right)$ OBDD variables [ Milner, 1980]. It has been recently shown in [Katz et al., 1999; Katz, 2001] how early quantification and variable interleaving in the OBDD can be used in order to reduce the number of required variables to $2 n$. Similarly, a naive implementation of the fixed-point expression with which the relation $\mathcal{C}$ is computed requires $4 n+2 n^{\prime}$ OBDD 
variables. We show how early quantification and variable interleaving can be used also here, reducing the number of required variables to $3 \gamma$, where $\gamma=\max \left\{n, n^{\prime}\right\}$.

Often, the designer is sufficiently familiar with the implementation and the specification to suspect that specific parts of the implementation are not covered by a specification. In such cases, it makes sense to replace the above described coverage algorithms by algorithms that get as input a set $M U T \subseteq W \times A P$ of mutations with respect to which coverage should be checked. A pair $\langle w, q\rangle \in M U T$ corresponds to the mutant implementation $\tilde{\mathcal{I}}_{w, q}$. Again, a naive algorithm checks the corresponding mutant implementations one by one, and is more complex than simulation in a factor of $|M U T|$. We show that our improved algorithms can be applied also in this case. The enumerative algorithm is more complex than simulation only in a factor of $\log |M U T|$, and the symbolic algorithm requires $3 \gamma$ variables, with $\gamma=\max \left\{n, n^{\prime},|M U T|\right\}$. In fact, the above described algorithms can be viewed as a special case where $M U T=W \times\{q\}$, for an observable signal $q$.

Due to the lack of space, we omit some proofs and technical details from this version. A full version can be found in the authors' URL.

\section{Preliminaries}

We model systems by labeled state transition graphs. Formally, a system $S$ is a tuple $S=\left\langle A P, W, R, W_{0}, L\right\rangle$, where $A P$ is a set of atomic propositions, $W$ is a set of states, $R \subseteq W \times W$ is a total transition relation, $W_{0}$ is a set of initial states, and $L: W \times A P \rightarrow\{$ true, false $\}$ is a labeling function that maps a state $w$ and an atomic proposition $p$ to the value of $p$ in $w$. We use $L(w)$ to denote the set $\{p: p \in A P$ and $L(w, p)=$ true $\}$. For a state $w$, we denote by $\operatorname{pre}(w)$ the set of direct predecessors of $w$ in the system, and by post $(w)$ the set of direct successors of $w$ in the system. Formally, $\operatorname{pre}(w)=\{v \in W: R(v, w)\}$, and $\operatorname{post}(w)=\{v \in W: R(w, v)\}$.

Consider an implementation $\mathcal{I}=\left\langle A P, W, R, W_{0}, L\right\rangle$, and a specification $\mathcal{S}=$ $\left\langle A P^{\prime}, W^{\prime}, R^{\prime}, W_{0}^{\prime}, L^{\prime}\right\rangle$. For technical convenience, we assume that $A P=A P^{\prime}$; thus, the implementation and the specification are defined over the same set of atomic propositions ${ }^{2}$. A binary relation $B \subseteq W \times W^{\prime}$ is a simulation (of $\mathcal{I}$ by $\mathcal{S}$ ) if for all $\left\langle w, w^{\prime}\right\rangle \in B$ the following conditions hold:

$$
1 L(w)=L^{\prime}\left(w^{\prime}\right) \text {. }
$$

2 For each $u$ such that $R(w, u)$ there exists $u^{\prime}$ such that $R^{\prime}\left(w^{\prime}, u^{\prime}\right)$ and $B\left(u, u^{\prime}\right)$.

It is easy to see that the union of two simulations is a simulation. Consequently, the maximal simulation between $\mathcal{I}$ and $\mathcal{S}$, denoted $\mathcal{B}$, is the union of all simulations of $\mathcal{I}$ by $\mathcal{S}$. We say that $w^{\prime} \in W^{\prime}$ simulates $w \in W$ if $\left\langle w, w^{\prime}\right\rangle \in \mathcal{B}$. We say that $\mathcal{S}$ simulates $\mathcal{I}$, or, equivalently, $\mathcal{I}$ is simulated by $\mathcal{S}$ (denoted $\mathcal{I} \leq \mathcal{S}$ ), if for every $w_{0} \in W_{0}$ there exists $w_{0}^{\prime} \in W_{0}^{\prime}$ that simulates $w_{0}$. Intuitively, it means that $\mathcal{S}$ has more behaviors than $\mathcal{I}$. In fact, every $\forall \mathrm{CTL}^{\star}$ formula that is satisfied in $\mathcal{S}$ is satisfied also in $\mathcal{I}$ [Browne et al., 1988; Grumberg and Long, 1994].

For the implementation $\mathcal{I}$, a state $w \in W$, and an atomic proposition $q \in A P$, the mutant implementation $\tilde{\mathcal{I}}_{w, q}=\left\langle A P, W, R, W_{0}, \tilde{L}_{w, q}\right\rangle$ is obtained from $\mathcal{I}$ by flipping the value of $q$ in $w$. Formally, $\tilde{L}_{w, q}(w, q)=\neg L(w, q)$, and for all $\langle v, p\rangle \neq\langle w, q\rangle$, we 
have $\tilde{L}_{w, q}(v, p)=L(v, p)$. Consider a specification $\mathcal{S}$ such that $\mathcal{I} \leq \mathcal{S}$ and an atomic proposition $q \in A P$. We say that $w$ is $q$-covered by $\mathcal{S}$ if $\tilde{\mathcal{I}}_{w, q} \mathbb{\mathcal { S }}$. Intuitively, $w$ is $q$-covered by $\mathcal{S}$ if flipping the value of $q$ in $w$ creates a behavior that is not permitted by $\mathcal{S}$.

\section{Enumerative Approach}

In this section we describe an efficient algorithm for computing the set of $q$-covered states. Our algorithm is based on the enumerative algorithm of Henzinger et al., 1995 for simulation computation. Consider an implementation $\mathcal{I}=\left\langle A P, W, R, W_{0}, L\right\rangle$ and a specification $\mathcal{S}=\left\langle A P, W^{\prime}, R^{\prime}, W_{0}^{\prime}, L^{\prime}\right\rangle$ such that $\mathcal{I} \leq \mathcal{S}$. Given $q \in A P$, we compute the set of states of $\mathcal{I}$ that are $q$-covered by $\mathcal{S}$. When $q$ is clear from the context, we omit it from our notations.

We first describe the algorithm of Henzinger et al. The maximal simulation relation $\mathcal{B} \subseteq W \times W^{\prime}$ is the set of all pairs $\left\langle w, w^{\prime}\right\rangle \in W \times W^{\prime}$ such that $w^{\prime}$ simulates $w$. That is, the labeling of $w$ is equal to the labeling of $w^{\prime}$, and for each successor $u$ of $w$ there is a successor $u^{\prime}$ of $w^{\prime}$ such that $u^{\prime}$ simulates $u$. By the definition, $\mathcal{B}$ is the greatest fixed point of the equation

$$
\mathcal{B}=\left\{\left\langle w, w^{\prime}\right\rangle: \mathcal{B}_{0}\left(w, w^{\prime}\right) \wedge \forall u \exists u^{\prime}:\left[R(w, u) \rightarrow R^{\prime}\left(w^{\prime}, u^{\prime}\right) \wedge \mathcal{B}\left(u, u^{\prime}\right)\right]\right\},
$$

where

$$
\mathcal{B}_{0}=\left\{\left\langle w \cdot w^{\prime}\right\rangle: L(w)=L^{\prime}\left(w^{\prime}\right)\right\} .
$$

An alternative way to compute the relation $\mathcal{B}$ is to compute, for each $w \in W$, the set $\operatorname{sim}(w)$ of states of $\mathcal{S}$ that simulate $w$. The algorithm of Henzinger et al. starts with the maximal possible candidate for a simulation set for each $w$, namely, the set of all states in $W^{\prime}$ with the same labeling, and it repeatedly reduces the sets until it reaches a fixed point: as long as there is a state $w$, a successor $u$ of $w$, and a state $w^{\prime} \in \operatorname{sim}(w)$ such that there is no successor of $w^{\prime}$ in $\operatorname{sim}(u)$, the set $\operatorname{sim}(w)$ should be reduced by removing $w^{\prime}$. A straightforward implementation of such a fixed-point calculation is presented in the procedure Schematic Similarity in Figure 1 and has time complexity $O\left(m m^{\prime} n^{2} n^{\prime}\right)$. Henzinger et al. improve this algorithm in the following way. For each $w \in W$, the algorithm maintains two sets of states: $\operatorname{oldsim}(w)$ and $\operatorname{sim}(w)$. The set $\operatorname{oldsim}(w)$ is the candidate for the simulation set for $w$ that was computed in the previous iteration, and $\operatorname{sim}(w)$ is the reduction of $\operatorname{old} \operatorname{sim}(w)$ that is computed in this iteration. A fixed-point is reached when $\operatorname{sim}(w)=\operatorname{oldsim}(w)$ for all $w \in W$, thus no further reduction is possible. The reduction of $\operatorname{sim}(w)$ is based on the same observation as in the straightforward algorithm: if a state $w^{\prime}$ is removed from the simulation set of $w$, then the predecessors of $w^{\prime}$ that have no other successors in the simulation set of $w$ should be removed from the simulation sets of predecessors of $w$. An important observation that leads to the complexity of $O\left(m^{\prime} n+m n^{\prime}\right)$ is that a state $w^{\prime}$ can be removed from the simulation set of $w$ at most once during the algorithm. In addition, the algorithm uses a data structure that allows to compute the size of $\operatorname{post}\left(v^{\prime}\right) \cap \operatorname{sim}(u)$ for all $v^{\prime} \in W^{\prime}$ and $u \in W$ in constant time. The efficient version of the algorithm is described in the procedure Efficient_Similarity in Figure 1.

The naive approach for coverage runs the algorithm of Henzinger et al. for $\tilde{\mathcal{I}}_{v, q}$, for all $v \in W$. The complexity of this is $n$ times the complexity of the algorithm of Henzinger et al, which is $O\left(\left(m^{\prime} n+m n^{\prime}\right) n\right)$. A better approach is to use the fact 


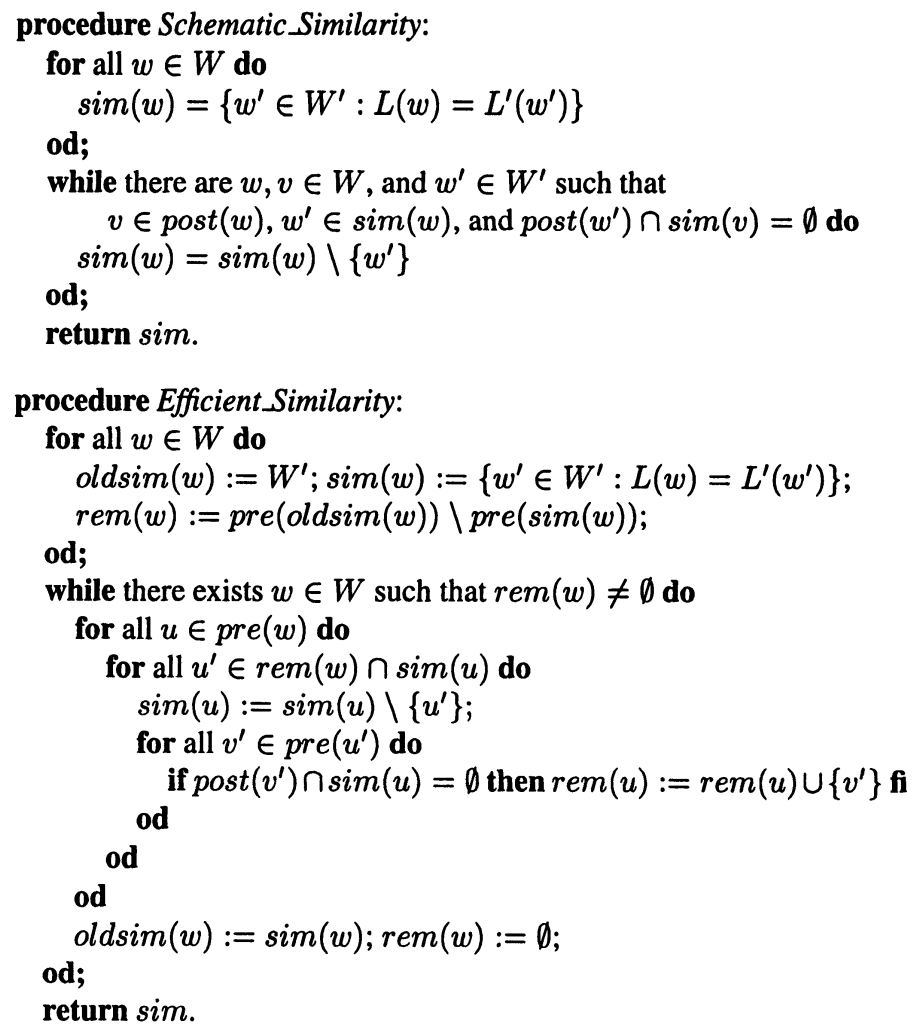

Figure 1. The similarity algorithm of Henzinger et al., 1995.

that for all $w, v \in W$, the implementations $\tilde{\mathcal{I}}_{w, q}$ and $\tilde{\mathcal{I}}_{v, q}$ differ only slightly (that is, only in labeling of two states). Thus there is room for hope that the simulation computation for $\tilde{\mathcal{I}}_{w, q}$ and $\tilde{\mathcal{I}}_{v, q}$ is also almost the same. In order to explain our approach, we introduce the notion of incomplete simulation. Let $X$ be the set of variables $\left\{x_{w}: w \in W\right\}$. For a subset of states $S \subseteq W$, the incomplete labeling function $L_{S}: W \times A P \rightarrow\{$ true, false $\} \cup X$ maps a pair $\langle w, p\rangle$ to $L(w, p)$ if $w \notin S$ or $p \neq q$, and to $x_{w}$ if $w \in S$ and $p=q$. As in the definition of $L$, we use $L_{S}(w)$ as a shortcut for the set $\left\{p: p \in A P\right.$ and $L_{S}(w, p)=$ true $\}$. For two states $w \in W$ and $w^{\prime} \in W^{\prime}$, and a set $S \subseteq W$, we say that $L_{S}(w)=L^{\prime}\left(w^{\prime}\right)$ if for every atomic proposition $p$, either $L_{S}(w, p)=L^{\prime}\left(w^{\prime}, p\right)$, or $L_{S}(w, p)$ is a variable. For a set of states $S \subseteq W$ we define the implementation $\mathcal{I}_{S}=\left\langle A P, W, R, W_{0}, L_{S}\right\rangle$ as $\mathcal{I}$ with the incomplete labeling function $L_{S}$. Let $\operatorname{sim}_{S}: W \rightarrow 2^{W^{\prime}}$ denote the maximal simulation relation from $\mathcal{I}_{S}$ to $\mathcal{S}$. Also, for $w \in W$, let $\widetilde{\operatorname{sim}} w: W \rightarrow 2^{W^{\prime}}$ denote the maximal simulation from $\tilde{\mathcal{I}}_{w, q}$ to $\mathcal{S}$. 
Consider a state $w \in W$ and two sets $S_{1} \subseteq S_{2} \subseteq W$. It is easy to see that $\operatorname{sim}_{S_{1}}(w) \subseteq \operatorname{sim}_{S_{2}}(w)$. Indeed, the set $\left\{w^{\prime}: w^{\prime} \in \bar{W}^{\prime}\right.$ and $\left.L_{S_{1}}(w)=L^{\prime}\left(w^{\prime}\right)\right\}$ is contained in the set $\left\{w^{\prime}: w^{\prime} \in W^{\prime}\right.$ and $\left.L_{S_{2}}(w)=L^{\prime}\left(w^{\prime}\right)\right\}$, and both simulation sets are computed from the above sets using the same monotonic fixed-point expression. In particular, when $S_{1}=\emptyset$, we have that $\operatorname{sim}(w) \subseteq \operatorname{sim}_{S}(w)$ for all $w \in W$ and $S \subseteq W$.

Let $S_{1} \subset S_{2} \subseteq W$ be two sets of states of $\mathcal{I}$. Assume that we have computed $\operatorname{sim}_{S_{2}}$ and now we wish to compute $\operatorname{sim}_{S_{1}}$. We claim that the computation of $\operatorname{sim}_{S_{1}}$ can be done using the algorithm of Henzinger et al. with the following modification: for each $w \in W$, we initialize the set $\operatorname{oldsim}_{S_{1}}(w)$ to $\operatorname{sim}_{S_{2}}(w)$ and the set $\operatorname{sim}_{S_{1}}(w)$ to $\operatorname{sim}_{S_{2}}(w) \cap\left\{w^{\prime} \in W^{\prime}: L^{\prime}\left(w^{\prime}\right)=L_{S_{1}}(w)\right\}$. In other words, in the initialization of oldsim ${ }_{S_{1}}(w)$ and $\operatorname{sim}_{S_{1}}(w)$, we intersect the sets initialized in [ Henzinger et al., 1995] with the set $\operatorname{sim}_{S_{2}}(w)$. Formally, consider the procedure Efficient_Incomplete-Similarity described in Figure 2. The procedure gets two parameters: $S \subseteq W$, and a simulation function $\operatorname{sim}^{\prime}: W \rightarrow 2^{W^{\prime}}$. It differs from Efficient_Similarity only in the initialization stage: when $S$ is not a singleton, the procedure computes the simulation relation by initializing sim and oldsim with respect to $L_{S}$ and $\operatorname{sim}^{\prime}$. When $S=\{w\}$, the procedure computes the simulation relation by initializing sim and oldsim with respect to $\tilde{L}_{w, q}$ and $\operatorname{sim}^{\prime}$.

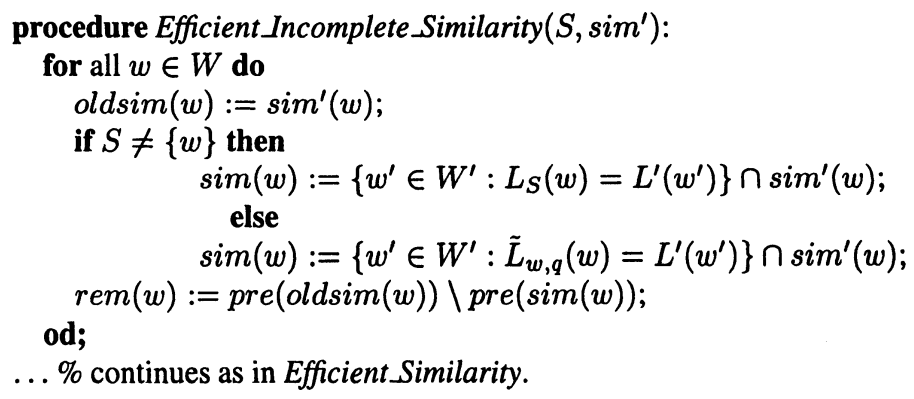

Figure 2. Incomplete similarity algorithm

When $\operatorname{sim}^{\prime}=\operatorname{sim}_{S^{\prime}}$ for $S \subseteq S^{\prime}$, the tighter initialization does not effect the correctness of the procedure. Formally, we have the following.

Lemma 1 Let $S_{1} \subseteq S_{2}$ be two subsets of $W$.

- If $\left|S_{1}\right|>1$, then Efficient_Incomplete_Similarity $\left(S_{1}, \operatorname{sim}_{S_{2}}\right)$ returns $\operatorname{sim}_{S_{1}}$ (from $\mathcal{I}_{S_{1}}$ to $\left.\mathcal{S}\right)$.

- If $S_{1}=\{v\}$, then Efficient_Incomplete_Similarity $\left(S_{1}, \operatorname{sim}_{S_{2}}\right)$ returns $\widetilde{\operatorname{sim}}_{v}$ (from $\tilde{\mathcal{I}}_{v, q}$ to $\mathcal{S}$ ).

We are now ready to describe our algorithm. The algorithm is based on a stepwise computation of the simulation relation $\operatorname{sim}$ from $\mathcal{I}$ to $\mathcal{S}$. In the first step, we compute 
incomplete simulation $\operatorname{sim}_{W}$ from $\mathcal{I}_{W}$ to $\mathcal{S}$. Note that $\operatorname{sim}_{W}$ refers to the labels of all the atomic propositions except $q$, and is very likely (a likelihood that increases for large sets of atomic propositions) to be much tighter than the initial candidate used in Efficient Similarity. Consider a partition of $W$ into two equal sets, $W_{1}$ and $W_{2}$. Our algorithm essentially works as follows. For all the mutant implementations $\tilde{\mathcal{I}}_{w, q}$ such that $w \in W_{1}$, the states in $W_{2}$ maintain their original labeling. Therefore, we start by computing incomplete simulation from $\mathcal{I}_{W_{1}}$ to $\mathcal{S}$; that is, we compute simulation with a labeling function that does not rely on the values of $q$ in states in $W_{1}$. We end up with the function $\operatorname{sim}_{W_{1}}$. Then, we continue and partition the set $W_{1}$ into two equal sets, $W_{11}$ and $W_{12}$, and calculate incomplete simulation from $\mathcal{I}_{W_{11}}$ to $\mathcal{S}$. The important point is that we can start the computation of $\operatorname{sim}_{W_{11}}$ from $\operatorname{sim}_{W_{1}}$. Thus, we have to reduce the current candidate only with respect to information that involves the values of $q$ in $W_{12}$. In a similar way, we compute incomplete simulation from $\mathcal{I}_{W_{2}}$ by $\mathcal{S}$, and then partition the set $W_{2}$ into two equal sets $W_{21}$ and $W_{22}$, and compute incomplete simulation from $\mathcal{I}_{W_{21}}$ and $\mathcal{I}_{W_{22}}$ to $\mathcal{S}$. Here, we can start the computation of $\operatorname{sim}_{W_{21}}$ and $\operatorname{sim}_{W_{22}}$ from $\operatorname{sim}_{W_{2}}$. Thus, as we go deeper in the recursion described above, we perform less work. The depth of the recursion is bounded by $\log |W|$. As we shall analyze exactly below, the work in depth $i$ amounts in average to performing $1 / 2^{i}$ of the work required for computing the full simulation relation. Hence the $O\left(\left(m^{\prime} n+m n^{\prime}\right) \log n\right)$ complexity.

In the full version, we describe the algorithm and analyze its complexity in detail.

Mutant vector. As discussed in the introduction, often it is helpful to allow the designer to specify a set $M U T \subseteq W \times A P$ of mutations with respect to which coverage should be checked. Each pair $\langle w, q\rangle$ in $M U T$ represents the mutant implementation $\tilde{\mathcal{I}}_{w, q}$. The algorithm above can be viewed as a special case where $M U T=W \times\{q\}$. It is easy to extend the algorithm to the more general case as follows. Given $M U T$, let $X_{M U T}=\left\{x_{w, q}:\langle w, q\rangle \in M U T\right\}$ be a set of variables that correspond to possible mutations. The incomplete labeling function $L_{M U T}$ agrees with $L$ for all $\langle w, q\rangle \notin M U T$ and is $x_{w, q}$ for $\langle w, q\rangle \in M U T$. In each step we randomly divide the set of variables into two equal subsets, and assign half of the variables their original values. Then we compute the incomplete simulation for this assignment. When the set of variables becomes a singleton $\left\{x_{w, q}\right\}$, we assign to $x_{w, q}$ the complementary value (that is, flip the value of $q$ in $w$ ), and compute the simulation function from the mutant implementation $\tilde{\mathcal{I}}_{w, q}$ to $\mathcal{S}$. The number of steps in the algorithm is $O(\log |M U T|)$. By the same considerations detailed for the special case, this leads to an average time complexity of $O\left(\left(m^{\prime} n+m n^{\prime}\right) \log |M U T|\right)$.

\section{Symbolic Approach}

In this section we present an algorithm that symbolically computes the set of $q$ covered states. Note that the naive approach, which executes a symbolic algorithm $|W|$ times for all mutant implementations, is no longer symbolic, as it requires explicit enumeration of the state space. The algorithm we present in this section is symbolic, 
and it computes the relation $\mathcal{C}$ that is defined as follows.

$$
\mathcal{C}=\left\{\left\langle w, v, w^{\prime}\right\rangle: w, v \in W, w^{\prime} \in W^{\prime}, \text { and } w^{\prime} \text { simulates } w \text { in } \tilde{\mathcal{I}}_{v, q}\right\} .
$$

Then, $v$ is $q$-covered by $\mathcal{S}$ if there is $w_{0} \in W_{0}$ such that for all $w_{0}^{\prime} \in W_{0}^{\prime}$, we have $\left\langle w_{0}, v, w_{0}^{\prime}\right\rangle \notin \mathcal{C}$. Several symbolic simulation algorithms are described in the literature [McMillan, 1993; Henzinger et al., 1995; Katz et al., 1999]. We build our coverage algorithm on top of the straightforward symbolic implementation of Milner's fixed-point expression for simulation (see $\mathcal{B}$ in Section 2). The reason for this, as we elaborate below, is the small number of OBDD variables that are needed in this approach. It is not hard to see that the relation $\mathcal{C}$ is the greatest fixed point of the following equation.

$$
\mathcal{C}=\left\{\left\langle w, v, w^{\prime}\right\rangle: \mathcal{C}_{0}\left(w, v, w^{\prime}\right) \wedge \forall u \exists u^{\prime}:\left[R(w, u) \rightarrow R^{\prime}\left(w^{\prime}, u^{\prime}\right) \wedge \mathcal{C}\left(u, v, u^{\prime}\right)\right]\right\},
$$

where $\mathcal{C}_{0}=\left\{\left\langle w, v, w^{\prime}\right\rangle: \tilde{L}_{v, q}(w)=L^{\prime}\left(w^{\prime}\right)\right\}$.

Thus, the calculation of $\mathcal{C}$ is very similar to that of $\mathcal{B}$, only that the state $v$ affects the labels that are compared in $\mathcal{C}_{0}$. The straightforward symbolic implementation of the above fixed point involves OBDDs with $6 n$ variables, where $n=\max \left(|W|,\left|W^{\prime}\right|\right)$. We show how to reduce the number of OBDD variables to $3 n$. In order to do so, we use early quantification and variable interleaving in the OBDDs. Both techniques are used in [Katz et al., 1999] (see also [Katz, 2001]) in order to reduce the number of OBDD variables required for computing the simulation relation $\mathcal{B}$ from $4 n$ to $2 n$.

We first explain the techniques in more detail. In early quantification, we try to push existential quantification inside in order to quantify out variables as soon as possible. Early quantification is traditionally used in conjunctive partitioning [Chen and Bryant, 1998; Yang, 1999] and is based on the property that sub-expressions can be moved out of the scope of an existential quantifier if they do not depend on any of the variables being quantified. In [Katz et al., 1999], early quantification is used for computing simulation as follows. Recall that $\mathcal{B}$ is the greatest fixed point of the expression

$$
\mathcal{B}=\left\{\left\langle w, w^{\prime}\right\rangle: \mathcal{B}_{0}\left(w, w^{\prime}\right) \wedge \forall u \exists u^{\prime}:\left[R(w, u) \rightarrow R^{\prime}\left(w^{\prime}, u^{\prime}\right) \wedge \mathcal{B}\left(u, u^{\prime}\right)\right]\right\},
$$

whose calculation involves OBDDs with $4 n$ variables. By early quantification of $u^{\prime}$, we get

$\mathcal{B}=\left\{\left\langle w, w^{\prime}\right\rangle: \mathcal{B}_{0}\left(w, w^{\prime}\right) \wedge \forall u\left[R(w, u) \rightarrow \exists u^{\prime}\right.\right.$ such that $\left.\left.R^{\prime}\left(w^{\prime}, u^{\prime}\right) \wedge \mathcal{B}\left(u, u^{\prime}\right)\right]\right\}$

A naive implementation of the new fixed-point involves OBDDs with $3 n$ variables. Indeed, the variables of $u$ are introduced only after these of $u^{\prime}$ are quantified out. In order to reduce the number of variables further, Katz et al. order the variables in the OBDDs so that the variables of a binary relation $f(x, y)$ are interleaved: the variables of $x$ are in the even levels of the OBDD for $f$ and these of $y$ are in the odd levels. Then, Katz et al. define two new operations on OBDD: comp and comp_odd. These operations compute "exist ... and" as one operation. Formally,

$$
\operatorname{comp}(f(x, y), g(x, z))=\exists x(f(x, y) \wedge g(x, z)),
$$

and

$$
\operatorname{comp\_ odd}(f(y, x), g(z, x))=\exists x(f(y, x) \wedge g(z, x))
$$


When the variables of $f$ and $g$ interleaved as described above, the implementation of comp and comp_odd can proceed in levels, where the corresponding element of $x$ is quantified simultaneously in the OBDDs of $f$ and $g$. The resulting OBDD refers to the variables in $y$ and $z$ only, so we stay with an OBDD with $2 n$ variables. For the detailed implementation of the operations see [Katz, 2001].

Now $\mathcal{B}$ can be calculated using only $2 n$ variables, by finding the greatest fixed point of the expression

$\mathcal{B}=\left\{\left\langle w, w^{\prime}\right\rangle: \mathcal{B}_{0}\left(w, w^{\prime}\right) \wedge \neg c o m p \_o d d\left(R(w, u), \neg\right.\right.$ comp_odd $\left.\left.\left(R^{\prime}\left(w^{\prime}, u^{\prime}\right), \mathcal{B}\left(u, u^{\prime}\right)\right)\right)\right\}$.

Using the same ideas, we apply early quantification to the fixed-point expression for $\mathcal{C}$ and get

$$
\mathcal{C}=\left\{\left\langle w, v, w^{\prime}\right\rangle: \mathcal{C}_{0}\left(w, v, w^{\prime}\right) \wedge \forall u\left[R(w, u) \rightarrow \exists u^{\prime}: R^{\prime}\left(w^{\prime}, u^{\prime}\right) \wedge \mathcal{C}\left(u, v, u^{\prime}\right)\right]\right\} .
$$

Then, we define two new operations on OBDDs, (3:0)-c and (3:2)-c, as follows.

$$
\text { (3:0)-c(f(x,y),g(x,z,u)) }=\exists x(f(x, y) \wedge g(x, z, u)) \text {, }
$$

and

$$
\text { (3:2)-c(f(y,x),g(u,z,x)) }=\exists x(f(y, x) \wedge g(u, z, x)) .
$$

The operations assume that the variables in the OBDDs of a ternary relation $g(x, z, u)$ are interleaved: the variables of $x$ are in the 0 mod 3 levels, these of $z$ are in the $1 \bmod 3$ levels, and these of $u$ are in the 2 mod 3 levels. Then, as in the case of binary relations, the existential quantification can be done in levels, with one pass on the OBDDs of $f$ and $g$, and with only $3 n$ variables.

Then, the relation $\mathcal{C}$ is the greatest fixed point of the expression

$\mathcal{C}=\left\{\left\langle w, v, w^{\prime}\right\rangle: \mathcal{C}_{0}\left(w, v, w^{\prime}\right) \wedge \neg(3: 2)-c\left(R(w, u), \neg(3: 2)-c\left(R^{\prime}\left(w^{\prime}, u^{\prime}\right), \mathcal{C}\left(u, v, u^{\prime}\right)\right)\right)\right\}$,

which can be calculated with $3 n$ variables.

Mutant vector. Given a mutant vector $M U T \subseteq W \times A P$, the above algorithm can be adjusted to calculate symbolically which of the mutants are covered by the specification. Note that $M U T$ can be given in some symbolic way (in particular, the above algorithm handles the case where $M U T=W \times\{q\}$ for some observable signal $q$ ), in which case it may be crucial to avoid an explicit enumeration of its members.

For a mutant $\lambda=\langle w, q\rangle \in M U T$, let $\tilde{L}_{\lambda}$ be the labeling function with $q$ flipped in $w$, and let $\tilde{\mathcal{I}}_{\lambda}$ be the corresponding mutant implementation. We would like to calculate the relation

$$
\mathcal{C}=\left\{\left\langle w, \lambda, w^{\prime}\right\rangle: w \in W, \lambda \in M U T, w^{\prime} \in W^{\prime}, \text { and } w^{\prime} \text { simulates } w \text { in } \tilde{\mathcal{I}}_{\lambda}\right\} .
$$

The relation $\mathcal{C}$ is the greatest fixed point of the expression

$$
\mathcal{C}=\left\{\left\langle w, \lambda, w^{\prime}\right\rangle: \mathcal{C}_{0}\left(w, \lambda, w^{\prime}\right) \wedge \forall u \exists u^{\prime}:\left[R(w, u) \rightarrow R^{\prime}\left(w^{\prime}, u^{\prime}\right) \wedge \mathcal{C}\left(u, \lambda, u^{\prime}\right)\right]\right\},
$$

where

$$
\mathcal{C}_{0}=\left\{\left\langle w, \lambda, w^{\prime}\right\rangle: \lambda \in M U T \text { and } \tilde{L}_{\lambda}(w)=L^{\prime}\left(w^{\prime}\right)\right\}
$$


As in the algorithm above, we can rewrite $\mathcal{C}$ so that the quantification on $u^{\prime}$ is pushed inside. Unlike in the algorithm above, here the members of the triplets are not only states of $\mathcal{I}$ and $\mathcal{S}$ but also members of $M U T$. Accordingly, we define $n=\max \left\{|W|,\left|W^{\prime}\right|,|M U T|\right\}$. Now, we can interleave the variables of $w, \lambda$, and $w^{\prime}$ as in the algorithm above, and calculate $\mathcal{C}$ as the greatest fixed point of the expression

$\mathcal{C}=\left\{\left\langle w, \lambda, w^{\prime}\right\rangle: \mathcal{C}_{0}\left(w, \lambda, w^{\prime}\right) \wedge \neg(3: 2)-c\left(R(w, u), \neg(3: 2)-c\left(R^{\prime}\left(w^{\prime}, u^{\prime}\right), \mathcal{C}\left(u, \lambda, u^{\prime}\right)\right)\right)\right.$, which can be calculated with $3 n$ variables.

\section{Discussion}

We defined mutation-based coverage for implementations and specifications given by labeled state-transition graphs and described two algorithms for computing the set of states covered by the specification. The general idea of the algorithms is similar to the idea used in [Chockler et al., 2001b] for mutation-based coverage in model-checking. The technical details, however, are different and nontrivial: in the enumerative algorithm, the overlaps between the mutant implementations lead to tighter candidates for simulation to start with ${ }^{3}$. In the symbolic approach, we addressed the problem of reducing the number of variables in the OBDDs involved, an issue that is not referred to in [Chockler et al., 2001b], where a naive implementation requires only $2 n$ variables. In addition, we show how the ideas in [Chockler et al., 2001b] can be extended to handle a given vector of mutations.

Our work brings together the "mutant-based approach" of [Hoskote et al., 1999] and the "simulation approach" of [Katz et al., 1999]. As in [Hoskote et al., 1999], coverage is measured with respect to mutant implementations. As in [Katz et al., 1999], conformance to specification is checked by simulation. In the full version, we discuss the relation between the two approaches in detail and show that the criteria defined in [Katz et al., 1999] for measuring coverage are orthogonal to our coverage measure.

\section{Notes}

1. The algorithm in Henzinger et al., 1995 is presented for the computation of the simulation relation in the same system, yet it can be easily adjusted to compute the simulation between an implementation and its specification.

2. By replacing a set $L(w) \in 2^{A P}$ by the set $L(w) \cap A P^{\prime}$, all our algorithms and results are valid also for the case $A P \supset A P^{\prime}$.

3. Alternatively, one could have followed the game-theoretic approach to simulation, show how incomplete simulation shrinks the game graph, and apply results from circuit complexity about shrinkage. This, less direct, approach would have been very similar to the approach taken in [Chockler et al., 2001b].

\section{References}

Abadi, M. and Lamport, L. (1991). The existence of refinement mappings. TCS, 82(2):253-284.

Beaty, D. and Bryant, R. (1994). Formally verifying a microprocessor using a simulation methodology. In 31st DAC, pp. 596-602. IEEE Comp. Soc.

Beer, I., Ben-David, S., Eisner, C., and Rodeh, Y. (1997). Efficient detection of vacuity in ACTL formulas. In 9th CAV, LNCS 1254, pp. 279-290. 
Bergmann, J. and Horowitz, M. (1999). Improving coverage analysis and test generation for large designs. In IEEE ICCAD, pp. 580-584.

Browne, M., Clarke, E., and Grumberg, O. (1988). Characterizing finite Kripke structures in propositional temporal logic. TCS, 59:115-131.

Chen, Y. A. and Bryant, R. (1998). Verification of floating point adders. In 10th CAV, LNCS 1427, pp. 488-499.

Chockler, H., Kupferman, O., Kurshan, R., and Vardi, M. (2001a). A practical approach to coverage in model checking. In 13th CAV, LNCS 2102, pp. 66-78.

Chockler, H., Kupferman, O., and Vardi, M. (2001b). Coverage metrics for temporal logic model checking. In 7th TACAS, LNCS 2031, pp. 528 - 542.

Clarke, E.M., Grumberg O., and Peled D. (1999). Model Checking. MIT Press.

Cleaveland, R., Parrow, J., and Steffen, B. (1993). The concurrency workbench: A semanticsbased tool for the verification of concurrent systems. ACM TOPLAS, 15:36-72.

Dill, D. (1998). What's between simulation and formal verification? In 35st DAC, pp. 328-329.

Fallah, F., Ashar, P., and Devadas, S. (1999). Simulation vector generation from HDL descriptions for observability enhanced-statement coverage. In 36th DAC, pp. 666-671.

Grumberg, O. and Long, D. (1994). Model checking and modular verification. ACM TOPLAS, 16(3):843-871.

Henzinger, M., Henzinger, T., and Kopke, P. (1995). Computing simulations on finite and infinite graphs. In 36th FOCS, pp. 453-462.

Ho, R. and Horowitz, M. (1996). Validation coverage analysis for complex digital designs. In ICCAD, pp. 146-151.

Hoskote, Y., Kam, T., Ho, P.-H., and Zhao, X. (1999). Coverage estimation for symbolic model checking. In 36th DAC, pp. 300-305.

Katz, S. (2001). Techniques for increasing coverage of formal verification. M.Sc. Thesis, The Technion, Israel.

Katz, S., Geist, D., and Grumberg, O. (1999). "Have I written enough properties ?" a method of comparison between specification and implementation. In 10th CHARME, LNCS 1703, pp. 280-297.

Kupferman, O. and Vardi, M. (1999). Vacuity detection in temporal model checking. In 10th CHARME, LNCS 1703, pp. 82-96.

Kurshan, R. (1994). Computer Aided Verification of Coordinating Processes. Princeton Univ. Press.

Lam, S. and Shankar, A. (1984). Protocol verification via projection. IEEE TSE, 10:325-342.

Lamport, L. (1983). Specifying concurrent program modules. ACM TOPLAS, 5:190-222.

Lynch, N. (1996). Distributed algorithms. Morgan Kaufmann.

Lynch, N. A. and Tuttle, M. (1987). Hierarchical correctness proofs for distributed algorithms. In 6th PODC, pp. 137-151.

McMillan, K. (1993). Symbolic Model Checking. Kluwer Academic Publishers.

Meyer, A. and Stockmeyer, L. (1972). The equivalence problem for regular expressions with squaring requires exponential time. In 13th IEEE SSAT, pp. 125-129.

Milner, R. (1971). An algebraic definition of simulation between programs. In 2nd IJCAI, pp. 481-489. British Computer Society.

Milner, R. (1980). A Calculus of Communicating Systems, LNCS 92. Springer Verlag, Berlin.

Yang, B. (1999). Optimizing Model Checking Based on BDD Characterization. $\mathrm{PhD}$ thesis, School of Computer Science, Carnegie Mellon University. 